\title{
SPREAD: a high-resolution daily gridded precipitation dataset for Spain
} - an extreme events frequency and intensity overview

Roberto Serrano-Notivoli et al.

Correspondence to: Roberto Serrano-Notivoli (rs@unizar.es)

The copyright of individual parts of the supplement might differ from the CC BY 3.0 License. 


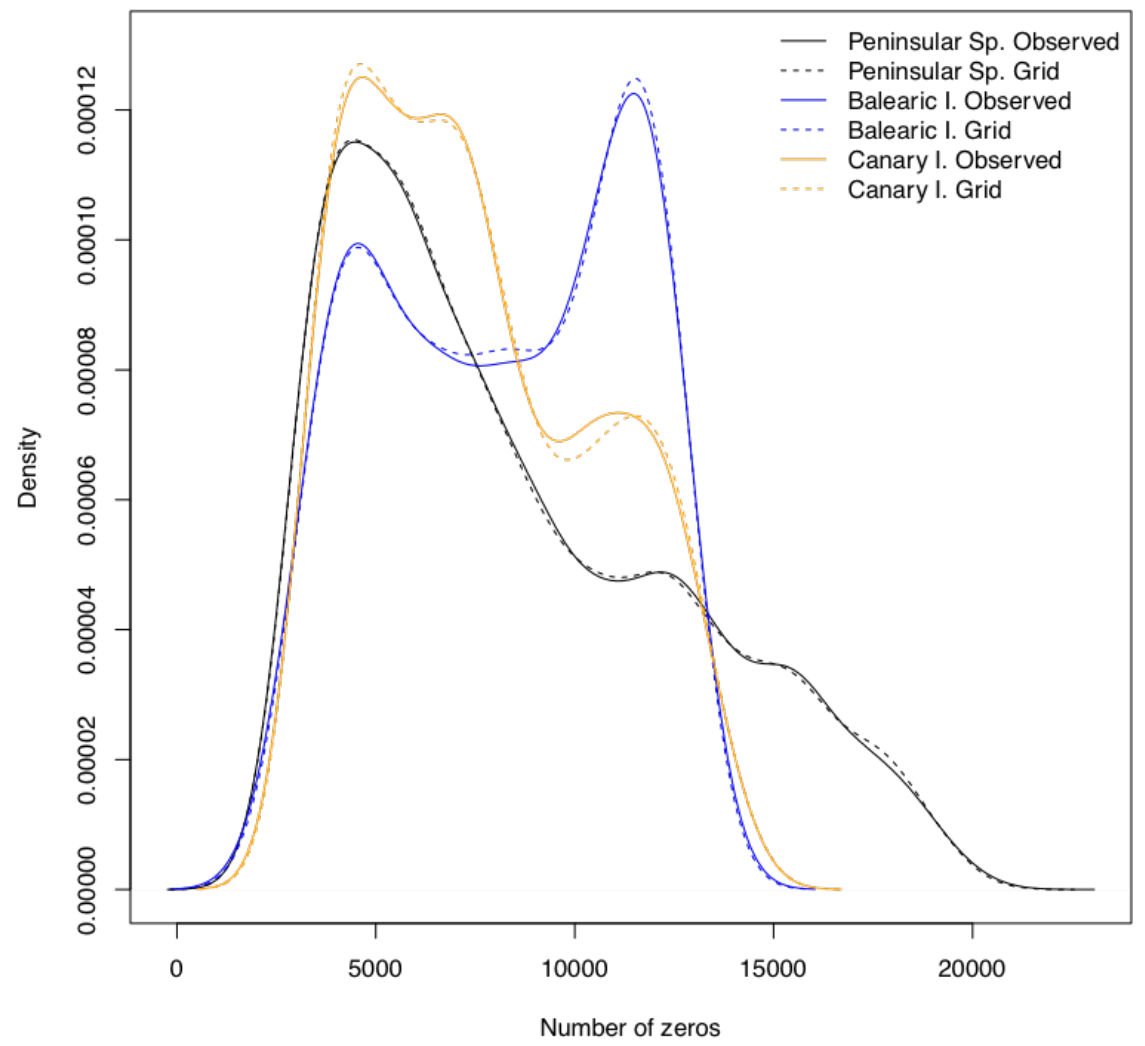

Figure S1: Comparison between number of observed (solid line) and estimated (dashed line) number of zeros in peninsular Spain (black lines), Balearic Islands (blue lines) and Canary Islands (orange lines). Kernel density curves. 

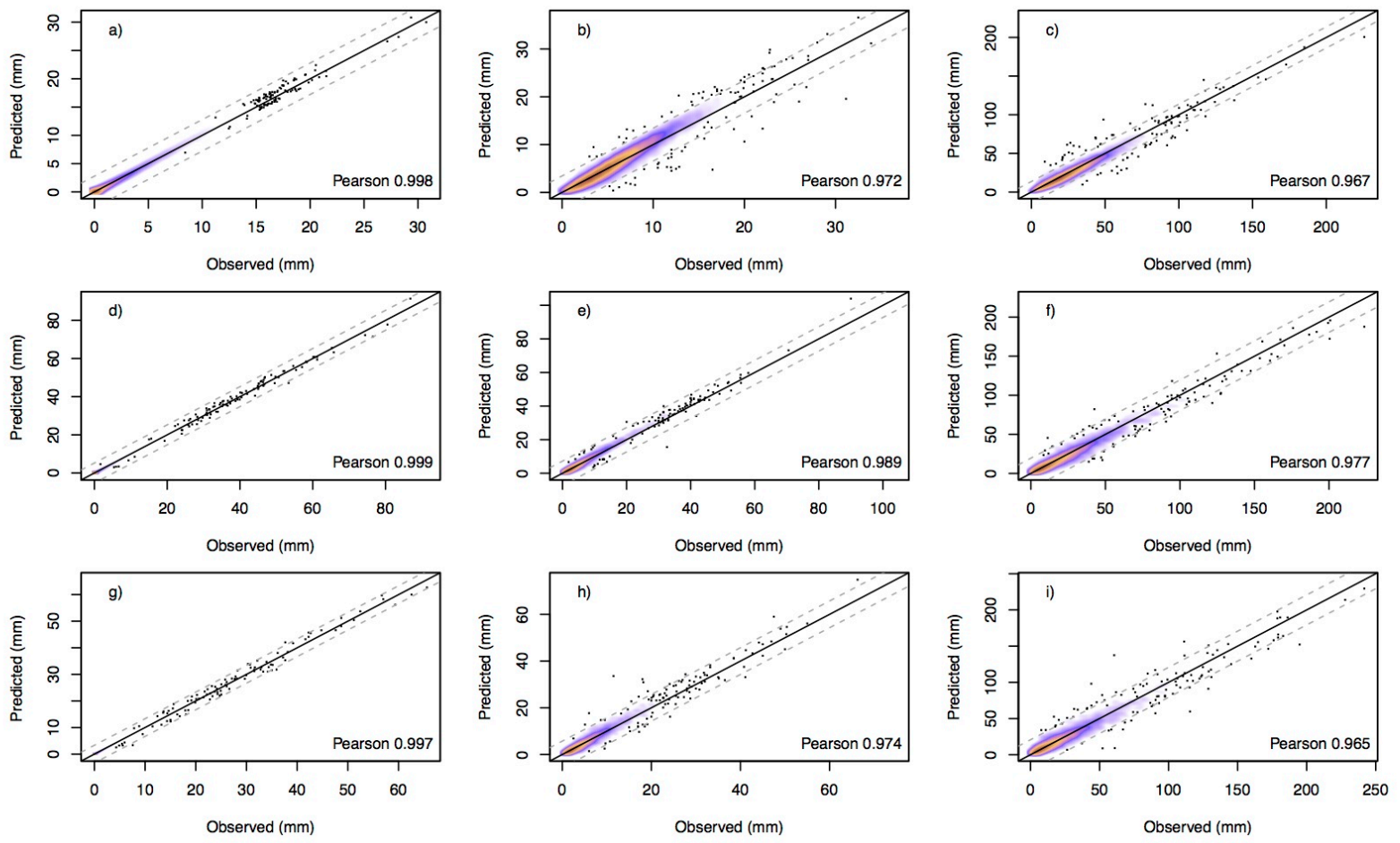

Figure S2: Scatterplots and Pearson correlation coefficients between observations and estimations in peninsular Spain (upper line, a b c), Balearic Islands (mid line $\mathrm{d}$ e $\mathrm{f}$ ) and Canary Islands (bottom line $\mathrm{g} \mathrm{h}$ i). Dots represent days and colours indicate the density. Daily precipitation mean (left column a d g); daily precipitation medians in wet days (central column b e h) and daily precipitation over 95 th percentile (right column $\mathrm{c} f$ i) are shown. 
Table S1: Leave-one-out cross-validation (LOO-CV) statistics showing the goodness of fit between observations and estimations of daily precipitation separated by deciles (D). IP: Iberian Peninsula; BI: Balearic Islands; CI: Canary Islands; MAE: Mean Absolute Error; ME: Mean Error; \%OBS: Percentage of observed precipitation; \%PRE: Percentage of predicted precipitation. Results were constrained to 2 decimal places.

\begin{tabular}{|c|c|c|c|c|c|c|c|c|c|c|c|}
\hline & & D1 & D2 & D3 & D4 & D5 & D6 & D7 & D8 & D9 & D10 \\
\hline \multirow{5}{*}{ IP } & Range & $\begin{array}{l}>0 \\
\text { to } \\
1.00\end{array}$ & $\begin{array}{l}1.00 \\
\text { to } \\
2.00\end{array}$ & $\begin{array}{l}2.00 \\
\text { to } \\
3.00\end{array}$ & $\begin{array}{l}3.00 \\
\text { to } \\
4.40\end{array}$ & $\begin{array}{l}4.40 \\
\text { to } \\
6.00\end{array}$ & $\begin{array}{l}6.00 \\
\text { to } \\
8.00\end{array}$ & $\begin{array}{l}8.01 \\
\text { to } \\
11.00\end{array}$ & $\begin{array}{l}11.00 \\
\text { to } \\
15.00\end{array}$ & $\begin{array}{l}15.01 \\
\text { to } \\
23.20\end{array}$ & $\begin{array}{l}23.21 \\
\text { to } \\
817.00\end{array}$ \\
\hline & $\%$ OBS & 0.70 & 1.70 & 2.40 & 3.70 & 5.70 & 6.80 & 10.20 & 12.10 & 18.80 & 38.00 \\
\hline & \%PRE & 2.60 & 3.70 & 4.00 & 5.20 & 7.10 & 7.80 & 10.70 & 11.80 & 17.00 & 30.10 \\
\hline & ME & 1.75 & 1.85 & 1.80 & 1.62 & 1.39 & 1.01 & 0.53 & -0.35 & -1.81 & -7.84 \\
\hline & MAE & 1.91 & 2.38 & 2.74 & 3.02 & 3.44 & 3.89 & 4.52 & 5.33 & 6.86 & 13.25 \\
\hline \multirow{6}{*}{ BI } & & $>0$ & 1.10 & 2.10 & 3.10 & 4.30 & 6.10 & 8.20 & 11.40 & 16.20 & 25.70 \\
\hline & Range & $\begin{array}{l}\text { to } \\
1.00\end{array}$ & $\begin{array}{l}\text { to } \\
2.00\end{array}$ & $\begin{array}{l}\text { to } \\
3.00\end{array}$ & $\begin{array}{l}\text { to } \\
4.20\end{array}$ & $\begin{array}{l}\text { to } \\
6.00\end{array}$ & $\begin{array}{l}\text { to } \\
8.10\end{array}$ & $\begin{array}{l}\text { to } \\
11.30\end{array}$ & $\begin{array}{l}\text { to } \\
16.10\end{array}$ & $\begin{array}{l}\text { to } \\
25.60\end{array}$ & $\begin{array}{l}\text { to } \\
304.00\end{array}$ \\
\hline & $\% \mathrm{OBS}$ & 0.60 & 1.60 & 2.20 & 3.00 & 5.20 & 5.90 & 9.00 & 12.50 & 18.80 & 41.20 \\
\hline & \%PRE & 1.90 & 2.80 & 3.10 & 3.80 & 6.10 & 6.50 & 9.40 & 12.50 & 17.90 & 36.00 \\
\hline & ME & 1.18 & 1.17 & 1.12 & 1.01 & 0.92 & 0.77 & 0.54 & 0.03 & -0.84 & -5.39 \\
\hline & MAE & 1.36 & 1.74 & 2.06 & 2.38 & 2.81 & 3.32 & 3.93 & 4.77 & 6.35 & 12.43 \\
\hline \multirow{5}{*}{ CI } & Range & $\begin{array}{l}>0 \\
\text { to } \\
0.80\end{array}$ & $\begin{array}{l}0.81 \\
\text { to } \\
1.50\end{array}$ & $\begin{array}{l}1.52 \\
\text { to } \\
2.50\end{array}$ & $\begin{array}{l}2.56 \\
\text { to } \\
3.50\end{array}$ & $\begin{array}{l}3.53 \\
\text { to } \\
5.00\end{array}$ & $\begin{array}{l}5.05 \\
\text { to } \\
7.00\end{array}$ & $\begin{array}{l}7.10 \\
\text { to } \\
10.00\end{array}$ & $\begin{array}{l}10.10 \\
\text { to } \\
15.00\end{array}$ & $\begin{array}{l}15.10 \\
\text { to } \\
25.40\end{array}$ & $\begin{array}{l}25.50 \\
\text { to } \\
590.00\end{array}$ \\
\hline & $\%$ OBS & 0.50 & 1.10 & 2.20 & 2.50 & 4.30 & 5.60 & 8.40 & 11.7 & 18.20 & 45.50 \\
\hline & \%PRE & 1.90 & 2.50 & 3.80 & 3.70 & 5.70 & 6.70 & 9.30 & 11.8 & 17.10 & 37.60 \\
\hline & ME & 1.46 & 1.52 & 1.47 & 1.43 & 1.44 & 1.13 & 0.92 & 0.15 & -1.15 & -8.21 \\
\hline & MAE & 1.60 & 2.00 & 2.39 & 2.86 & 3.47 & 4.03 & 4.97 & 6.00 & 8.31 & 17.52 \\
\hline
\end{tabular}


Table S2: Leave-one-out cross-validation (LOO-CV) statistics showing the goodness of fit between observations and estimations of daily precipitation separated by altitudes (m.a.s.l.). IP: Iberian Peninsula; BI: Balearic Islands; CI: Canary Islands; N: number of stations; MAE: Mean Absolute Error; ME: Mean Error; \%OBS: Percentage of observed precipitation; \%PRE: Percentage of predicted precipitation; RM: Ratio of Means; RSD: Ratio of Standard Deviations. Results were constrained to 2 decimal places.

\begin{tabular}{|c|c|c|c|c|c|c|c|c|c|c|c|}
\hline & & $0-100$ & $\begin{array}{l}>100- \\
300\end{array}$ & $\begin{array}{l}>\mathbf{3 0 0}- \\
\mathbf{5 0 0}\end{array}$ & $\begin{array}{l}>500- \\
700\end{array}$ & $\begin{array}{l}>700- \\
900\end{array}$ & $\begin{array}{l}>900- \\
1,100\end{array}$ & $\begin{array}{l}>1,100- \\
1,300\end{array}$ & $\begin{array}{l}>1,300 \\
-1,500\end{array}$ & $\begin{array}{l}>1,500- \\
2,000\end{array}$ & $>2,000$ \\
\hline \multirow{7}{*}{ IP } & $\mathbf{N}$ & 918 & 1,054 & 1,077 & 1,210 & 1,271 & 766 & 415 & 128 & 65 & 11 \\
\hline & \%OBS & 12.50 & 14.40 & 14.20 & 17.20 & 17.30 & 12.70 & 7.90 & 2.30 & 1.30 & 0.20 \\
\hline & \%PRE & 12.60 & 14.40 & 14.20 & 17.20 & 17.30 & 12.70 & 7.80 & 2.30 & 1.40 & 0.20 \\
\hline & $\mathbf{R M}$ & 1.00 & 0.99 & 0.99 & 0.99 & 0.99 & 0.99 & 0.98 & 1.01 & 1.06 & 1.07 \\
\hline & RSD & 0.95 & 0.96 & 0.96 & 0.96 & 0.97 & 0.96 & 0.96 & 1.03 & 1.10 & 1.19 \\
\hline & ME & 0.04 & -0.02 & -0.02 & -0.01 & 0.01 & 0.00 & -0.03 & 0.26 & 0.64 & 1.47 \\
\hline & MAE & 4.83 & 4.55 & 4.55 & 4.51 & 4.3 & 4.86 & 5.48 & 6.32 & 6.92 & 9.61 \\
\hline \multirow{7}{*}{ BI } & $\mathbf{N}$ & 128 & 93 & 20 & 4 & 2 & 1 & 0 & 0 & 0 & 0 \\
\hline & $\%$ OBS & 43.80 & 39.20 & 12.80 & 2.70 & 1.10 & 0.40 & & & & \\
\hline & \%PRE & 44.00 & 39.00 & 12.90 & 2.60 & 1.10 & 0.40 & & & & \\
\hline & $\mathbf{R M}$ & 1.02 & 0.99 & 1.03 & 0.84 & 1.09 & 1.43 & & & & \\
\hline & RSD & 1.00 & 0.96 & 0.97 & 0.96 & 0.99 & 1.34 & & & & \\
\hline & ME & 0.17 & -0.10 & 0.11 & -0.48 & 0.52 & 2.65 & & & & \\
\hline & MAE & 3.78 & 3.82 & 5.71 & 7.76 & 10.11 & 8.12 & & & & \\
\hline \multirow{7}{*}{ CI } & $\mathbf{N}$ & 78 & 96 & 79 & 70 & 40 & 37 & 13 & 9 & 9 & 10 \\
\hline & $\%$ OBS & 8.40 & 15.00 & 18.70 & 20.50 & 13.40 & 11.00 & 5.20 & 4.30 & 2.20 & 1.30 \\
\hline & $\%$ PRE & 8.80 & 15.20 & 18.40 & 20.40 & 13.20 & 10.90 & 5.20 & 4.20 & 2.30 & 1.40 \\
\hline & $\mathbf{R M}$ & 1.02 & 1.02 & 0.97 & 1.00 & 1.02 & 0.99 & 0.98 & 1.02 & 1.01 & 0.92 \\
\hline & RSD & 1.02 & 1.02 & 0.98 & 0.99 & 0.98 & 0.99 & 1.05 & 0.96 & 1.03 & 0.93 \\
\hline & ME & 0.39 & 0.17 & -0.15 & 0.00 & -0.13 & -0.15 & -0.02 & -0.16 & 0.48 & 1.85 \\
\hline & MAE & 4.49 & 4.18 & 5.28 & 5.91 & 5.94 & 5.95 & 6.26 & 5.09 & 5.48 & 14.28 \\
\hline
\end{tabular}

